\title{
Les dispositifs de FOAD dans les établissements d'enseignement supérieur : transfert ou intégration ?
}

\author{
Isabelle Bertrand \\ Cned-Direction générale \\ Téléport 2 \\ 2, boulevard Nicéphore Niepce \\ BP 80300 \\ F-86963 Futuroscope Chasseneuil cedex \\ isabelle.bertrand@cned.fr
}

RÉSUMÉ. L'importance croissante des technologies de l'information et de la communication remodèle les systèmes de formation dans l'enseignement supérieur et ceux la formation ouverte et à distance (FOAD). Cette évolution s'est traduite notamment par l'ouverture en 2001 de campus numériques en France à l'incitation de la direction de la technologie au ministère de la Recherche. La mise en place de l'un d'eux a soulevé la question suivante: Comment intégrer dans un établissement d'enseignement supérieur, et dans le métier d'enseignant, un dispositif de FOAD, conçu ailleurs? Nous proposons des repères bibliographiques relatifs à cette question examinée dans le contexte des campus numériques et de dispositifs concrets de FOAD, qui soulignent l'instabilité du concept de FOAD. Ces repères indiquent que les atouts techniques des dispositifs sont diversement exploités, que leur diffusion reste modeste et montrent que la FOAD dans l'enseignement supérieur n'est conceptuellement qu'émergente.

ABSTRACT. The growing impact of information and communication technology (ICT) on higher education and on open and distance learning (ODL) tends to reshape both. In the wake of this evolution, in 2001, the Direction de la technologie at the French Ministry of research encouraged the launching of a number of "digital campuses". During the setting up of one of those the following question arose "how can a higher education establishment and its academic staff integrate an ODL system which has been developped elsewhere?". The study of bibliographical references related to the context of the digital campuses back the idea that the ODL concept is not stable yet, that the technical assets of ODL systems are diversely taken into account and still sparsely used, and that ODL in higher education, even as a concept is only just emerging.

MOTS-CLÉS : dispositifs de FOAD, enseignement supérieur, campus numérique, transfert de dispositif de formation, approche pluridisciplinaire.

KEYWORDS: ODL system, higher education, digital campus, transfer of educational system, multidisciplinary approach. 
L'émergence de la société du savoir et l'évolution des technologies de l'information et de la communication (TIC) inscrivent un contexte où les systèmes d'enseignement et de formation sont amenés à évoluer. C'est tout particulièrement le cas pour l'enseignement supérieur et pour la formation à ouverte et à distance (FOAD).

Tandis que la FOAD passe de l'enseignement par correspondance à l'enseignement via internet, l'enseignement supérieur est engagé, quant à lui, à modulariser et à individualiser ses formations, à développer la «formation tout au long de la vie », pour répondre aux pressions des mondes économique, politique et professionnel.

Les TIC constituent un vecteur privilégié de cette rencontre de la FOAD et de l'enseignement supérieur. Certes, l'enseignement à distance existe au sein de l'enseignement supérieur depuis les années soixante par l'intermédiaire des Centres de télé-enseignement universitaires (CTEU), des formations dispensées par le Centre National des Arts et métiers (CNAM) ou par le Centre national d'enseignement à distance (Cned). Mais jusqu'à la fin des années 80, cette offre de formation supérieure et à distance était d'une importance quantitative réduite et concernait principalement un public en formation continue.

L'évolution du marché du travail, la modification des besoins de compétences, la croissance du nombre d'étudiants et le développement de la formation continue ont été autant de facteurs contribuant à ce qu'une politique globale en faveur d'un usage intensif des TIC soit mise en place par le gouvernement français.

Elle s'est traduite concrètement par la mise en chantier en 2001 de plusieurs campus numériques en France, à la suite d'un appel à projets lancé par les ministères de l'Éducation nationale et de la recherche.

C'est dans le cadre de la phase d'étude de faisabilité du projet campus numérique $\mathrm{n}^{\circ} 15$ qu'a été soulevée la question suivante: Comment intégrer dans un établissement d'enseignement supérieur, et dans le métier d'enseignant, un dispositif de formation ouvert et à distance, conçu ailleurs ?

Nous proposons, dans les pages qui suivent, quelques repères bibliographiques relatifs aux implications de cette question qui met en regard FOAD et enseignement supérieur :

- en évoquant d'abord le contexte des campus numériques en général et celui du campus $\mathrm{n}^{\circ} 15$ en particulier, pour illustrer l'intérêt et les enjeux soulevés par cette question ;

- en montrant que le concept de FOAD n'est pas stabilisé, que les angles d'analyse de la question sont variés et nécessitent des réponses pluridisciplinaires. Seront opposées la notion d'intégration des TIC à celle, plus industrielle, de transfert d'un dispositif conçu ailleurs, en étudiant des exemples concrets de dispositifs de FOAD dans l'enseignement supérieur. 
Cette étude met en lumière des dispositifs techniques, des mises en œuvre, et des modèles d'enseignement à distance très diversifiés.

Seront enfin présentés les principaux obstacles, d'ordre culturel, technique, et structurel rencontrés lors de la mise en place de dispositifs de FOAD.

Les références sur lesquelles s'appuie cet article sont des documents principalement recueillis via internet, des ouvrages issus des domaines disciplinaires abordés : sciences de l'information et de la communication, sciences de l'éducation, et FOAD.

Pour décrire la situation française en matière de FOAD dans l'enseignement supérieur, nous nous sommes servis de deux sources d'information qui nous ont parus complémentaires :

- la première témoigne de situations en cours. Ce sont les débats, les analyses produites par les associations fortement impliquées dans l'univers de la FOAD, que ce soit de manière générale (le Forum français pour la formation ouverte et à distance, FFFOD, l'association Algora formation ouverte et réseaux, par exemple), ou au sein de l'enseignement supérieur (par exemple, le Groupement pour l'enseignement supérieur sur mesure médiatisé, GEMME, la Fédération interuniversitaire de l'enseignement à distance, FIED) ;

- la seconde source est plus académique, et propose une mise en perspective de la FOAD à partir des disciplines auxquelles appartiennent les chercheurs. Ce sont des textes issus des sciences de l'éducation ou des sciences de l'information et de la communication, des analyses portant sur des thèmes tels que l'intégration, l'appropriation, les usages des TIC dans l'enseignement, ou bien encore sur l'industrialisation de l'enseignement.

\section{La FOAD dans l'enseignement supérieur : un paysage complexe}

\section{Contexte général}

La complexité du paysage de l'enseignement à distance au sein des établissements d'enseignement supérieur semble bien à l'image de l'enseignement supérieur lui-même.

$\mathrm{Au}$ sein de l'enseignement supérieur français se côtoient aussi bien des établissements offrant des cours par correspondance «classiques", que d'autres, développant des formations en ligne, ou une offre de «e-learning », notion qui reste à clarifier. 
Plus concrètement, selon l'association Algora, 35 à 40 universités (parmi les 90 universités françaises ${ }^{1}$ ) utilisent en 2000 des plates-formes de formation à distance $^{2}$, et plus de 280 cursus en ligne sont répertoriés en France ${ }^{3}$.

La rentrée 2000 a marqué un tournant concret dans l'intégration de l'utilisation des TIC avec un appel à projets des ministères de l'Éducation nationale et de la Recherche pour la création de campus numériques, avec une dotation de 18 millions de francs pour 2000, et de 40 pour 2001. En 2001, 27 projets ont été retenus pour une étude de faisabilité (ou niveau 1) et 39 sont en phase de réalisation (niveau 2) ${ }^{4}$.

Cet appel à projet s'est concrétisé dès 2001 par l'ouverture d'une dizaine de campus dans des champs disciplinaires variés : par exemple, l'économie et la gestion (CANEGE), la médecine (ESSQUAD), la chimie ou les sciences pour l'ingénieur (EDEN3). En 2002, 82 projets ont été présentés par plus de 200 établissements d'enseignement supérieur, en partenariat avec 64 établissements étrangers et les entreprises et associations des secteurs concernés. Parmi ceux-ci 36 ont été retenus pour recevoir un financement ${ }^{5}$.

L'appel à projets campus numériques français a le double objectif suivant: soutenir l'usage des TIC au sein des établissements d'enseignement supérieur français et développer une offre de formation à distance reposant sur l'implication forte de ces établissements. ${ }^{6}$

La mise en place de ces dispositifs a nécessité la mutualisation des moyens tant humains que techniques avec une identification parfois délicate des rôles joués par chacun des acteurs dans l'élaboration du dispositif. Par exemple, le campus numérique CANEGE (campus numérique en économie et gestion) réunit six établissements universitaires, le Cned et France Télécom. Le campus numérique FELICES (formation en ligne initiale dédiée à la formation des professeurs des lycées et collèges) repose sur l'association de dix établissements parmi lesquels figurent quatre universités, quatre instituts universitaires de formation des maîtres, l'INRP et le Cned. Enfin le campus EDEN3 (enseignement à distance en écoles d'ingénieur, $3^{\mathrm{e}}$ année) est soutenu par un consortium de sept établissements, dont quatre grandes écoles, une université et France Télécom.

\footnotetext{
1. Cf. le site de l'Éducation nationale, http://www.education.gouv.fr

2. Répertoire des formations 2001, L'étudiant.

3. «Quelles opportunités, quelles démarches, quelles formations ? », Agnès Arcier, DIGITIP, in Diplômées, mars 2001.

4. Pour une comparaison des projets retenus en 2000 et 2001, http://www.educnet.education.fr/superieur/campus2001.htm

5. Cf. http://www.educnet.education.fr

6. Averous M., Touzot G., (2002), Campus numériques : enjeux et perspectives pour la formation ouverte et à distance, http://www.formasup.fr
} 


\section{Des débats}

Cette mise en place suscite donc un certain nombre d'interrogations :

- quelles sont les transformations induites par de tels dispositifs, tant du point de vue organisationnel (mutualisation des compétences, partenariats entre établissements, gestion de logiques professionnelles différentes), qu'institutionnel (intégration et gestion d'acteurs nouveaux, revalorisation de l'enseignement), professionnel (émergence de nouvelles compétences), ou pédagogique (nouveaux modes d'apprentissages, de transmission de savoir)?

- comment se redistribuent les rôles des différents intervenants dans la chaîne de production et de la diffusion des connaissances?

Des débats sont aussi apparus, comme en témoigne une bibliographie féconde, sur les questions concernant :

- l'intégration des TIC dans le milieu de l'enseignement supérieur, par exemple à travers les usages observés ${ }^{7}$;

- les compétences ${ }^{8}$ indispensables à l'usage des TIC chez les enseignants et les étudiants. Parmi les travaux les plus «opérationnels» on note ceux issus de l'association Algora, et relatifs au milieu de la formation continue ;

- les pratiques pédagogiques des enseignants du supérieur ${ }^{9}$;

- l'interopérabilité, ainsi que les préoccupations ayant trait à la normalisation et la standardisation ${ }^{10}$.

Au-delà de la «vitrine» technologique qu'ils représentent, les campus numériques ont pour vertu de constituer de véritables laboratoires d'expérimentation susceptibles de mieux permettre de saisir les enjeux et les opportunités de l'utilisation des TIC dans les réseaux de formations supérieures.

7. Ologéanu R., (2000), Les usages de la visioconférence dans l'enseignement supérieur, sur le site http://www.educnet.education.fr/superieur/visio/visio1.htm

8. Haeuw F., Coulon A., (2001), L'évolution des compétences des formateurs dans les dispositifs de formation ouverte et à distance, sur le site :

http://www.oravep.asso.fr/synergie/pourtous/kiosque/publicat/ced/som.htm. C'est également un débat au sein des IUFM, comme en témoigne l'article de Drot-Delange B., Kuster Y., Tricot A. (2000), «La construction de quelques compétences TIC au cours de la seconde année d'IUFM. Analyse de deux cas », http://www.inrp.fr/Tecne/Rencontre/Tricot2.pdf

9. Colloque (2000), Apprendre et enseigner dans l'enseignement supérieur, à l'université de Paris X, sur le site http://webdev.uparis10.fr/SPUPX/sitesmgr.wwv_main.main?p_language=f\&p_cornerid=775 $\& p \_$currcornerid $=774 \& p \_$full $=1$

10. Arnaud M., Nardin R., Perriault J., Saillant J.-M., (2001), Rapport d'étape sur les pratiques en matière de normes et standards pour l'apprentissage en ligne, GEMME, sur le site http://gemme.univ-lyon1.fr/rapports.html 


\section{Le campus numérique 15}

C'est dans ce contexte que s'inscrit le projet du campus numérique $\mathrm{n}^{\circ} 15$, intitulé «Dispositif de formation à distance certifiée clef en main : expérimentation et évaluation d'un modèle permettant la démultiplication de formations dans le domaine des technologies de l'information », qui fut soutenu par quatre partenaires :

- le Groupement des écoles de télécommunication (GET), jouant le rôle de l'institution experte dans le domaine de formation, détentrice à la fois des contenus et de la certification ;

- le Centre national d'enseignement à distance (Cned), qui est l'opérateur de formation chargé de mettre à disposition la formation, de gérer les inscriptions des étudiants, d'effectuer le suivi de la formation ;

- l'institut ERIEE de l'Ecole des Mines d'Alès dans le rôle du client ;

- France Télécom qui est l'opérateur de télécommunication mettant à disposition de manière temporaire les réseaux nécessaires pour accéder aux informations fournies par l'opérateur de formation et au suivi pédagogique fourni par l'institution experte.

\section{La démultiplication de dispositifs FOAD}

Le projet initial des partenaires consistait à évaluer la pertinence sociale, pédagogique, technique et économique d'un modèle de dispositif de formation à distance certifié clefs en mains.

Ils décidèrent de privilégier l'axe social du projet, marquant ainsi un intérêt certain pour les conditions d'acceptabilité d'un dispositif de formation, pour les relations entre les acteurs de la formation, pour l'évolution des métiers que cela implique.

Les objectifs pédagogiques et didactiques initiaux consistaient :

- à définir puis tester un modèle de démultiplication de dispositif de formation permettant de répondre à un besoin de formation non satisfait,

- à étudier une offre de service de formation à distance adaptant les fonctionnalités et les outils de l'e-learning au contexte de service public de formation français,

- à expérimenter un dispositif de formation à distance mixte: support traditionnel et en ligne, accompagnement en présence et à distance.

Les «clients » potentiels d'un tel dispositif sont des organismes de formation initiale supérieure et le public visé est celui des étudiants de niveau bac+3 (année post-DUT) et bac+4 (en formation d'ingénieur).

L'exploration documentaire préalable a été complexe puisque la question du campus numérique $\mathrm{n}^{\circ} 15$ portait à la fois : 
- sur l'intégration des formations ouvertes et à distance au sein des établissements supérieurs,

- sur ces dispositifs en relation avec le métier d'enseignant,

- sur l'intégration de dispositifs conçu ailleurs, c'est-à-dire la possibilité de transferts de dispositifs de FOAD.

Le dernier point ne fait qu'illustrer une préoccupation latente dans le milieu des professionnels de la FOAD et la replace dans un contexte nouveau : quelles sont les conditions nécessaires pour pérenniser, capitaliser, assurer la transférabilité et généraliser l'impact des dispositifs de $\mathrm{FOAD}^{11}$ ?

L'intérêt de cette question réside dans le fait qu'elle cristallise les enjeux du moment concernant l'idéal d'un dispositif de FOAD générique, susceptible d'être adapté à n'importe quel contexte. Cet enjeu émane du milieu des producteurs de dispositifs de FOAD qui, dans le cadre de la formation professionnelle continue, pratiquent le transfert. La question, très polémique, met en exergue des prises de positions contradictoires, précisément parce qu'elle émerge au sein de l'enseignement supérieur, qui n'obéit pas à la même logique que celle des producteurs de dispositifs de FOAD.

\section{Difficultés conceptuelles}

L'exploration bibliographique de la question de l'intégration de dispositifs de FOAD, conçus ailleurs, au sein d'établissements d'enseignement supérieurs a soulevé en amont un certain nombre de difficultés.

\section{Un concept non stabilisé}

La première difficulté fut d'ordre conceptuel. En effet, les termes tels que «FOAD» ou «dispositifs de FOAD» se sont révélées comme étant des concepts pluridimensionnels, d'usage récent et pas encore stabilisés ${ }^{12}$.

Pour s'en convaincre il suffit de parcourir les multiples définitions produites autour de ces deux termes.

Elles sont institutionnelles : pour le ministère de l'Emploi et de la solidarité, Délégation générale à l'emploi et à la formation professionnelle, «Une formation ouverte et/ou à distance est un dispositif souple de formation organisé en fonction de besoins individuels ou collectifs (individus, entreprises, territoires). Elle

11. Cf. les débats développés au cours du colloque Téléform 2000, sur le site http://www.xcom.fr/teleform 2000

12. Cf. les débats occasionnés par ce terme au cours de la conférence de consensus de Chasseneuil, http://ressources.algora.org/reperes/comprendre/chasseneuil/index.asp 
comporte des apprentissages individualisés et l'accès à des ressources et compétences locales ou à distance. Elle n'est pas exécutée nécessairement sous le contrôle permanent d'un formateur. »

Elles sont également «savantes»: un dispositif FOAD $^{13}$ peut également recouvrir «Toute forme d'étude souple qui ne repose pas sur la présence physique de l'enseignant auprès de l'étudiant mais dont l'organisation et le contenu dépendent d'un organisme de formation. [... ] C'est une combinaison de systèmes formels de formation, un système formel de formation étant défini comme un ensemble de moyens matériels et humains, agencés en vue de faciliter un processus d'apprentissage. » A titre d'exemple de systèmes formels de formation, on note : les cours en salle, atelier, centres de ressources, cours par correspondance, cours télédiffusés (radio, téléphone, web), formation en ligne (EAO, simulateurs), communautés d'apprentissage, campus virtuels, classe virtuelles, télétutorat, télécours... chaque système relevant d'une conception et d'une école théorique différente ${ }^{14}$.

Cette pluralité de définitions est le résultat d'une évolution du concept de formation ouverte ${ }^{15}$, apparu dans les années 1990. A chaque nouvelle définition s'est ajoutée une nouvelle dimension. En 1992, la «formation ouverte », telle que la Commission des Communautés européennes la définit, s'est vue enrichie par la Délégation à la formation professionnelle (ministère du travail) d'une dimension supplémentaire, la flexibilité : «Les formations ouvertes sont des actions de formation s'appuyant en tout ou partie sur des apprentissages non présentiels en auto-formation ou avec tutorat, à domicile, dans l'entreprise ou en centre de formation. "

Enfin dans la définition produite par la conférence de consensus de Chasseneuil en 2000, aux notions d'ouverture et de flexibilité est associée celle de combinaison de situation d'apprentissage. C'est celle retenue par les divers acteurs impliqués dans le projet de campus numérique 15: «un dispositif organisé, finalisé, reconnu comme tel,

- qui prend en compte la singularité des personnes dans leurs dimensions individuelle et collective,

13. Blandin B., «Open and distance learning within the world of vocational training and lifelong learning », Part I, Open and distance learning, an overall survey at the beginning of 2000, texte présenté lors du colloque ODL Networking for Quality Learning à Lisbonne, les 19-20 et 21 juin 2000, $20 \mathrm{p}$.

14. Internet: nouveaux horizons pour la formation, étude ADAPT BIS-NAT/97/259 A, 1998/1999.

15. «Former et échanger par les réseaux», Geneviève Lameul, avril 2000, www.iufm.fr/f_tic.htm, «La FOAD, de quoi s'agit-il ? De la définition au marché », Philippe Morin, in Formation à distance et technologies de la communication, Actes du 14 décembre 2000. 
- et repose sur des situations d'apprentissage complémentaires et plurielles en termes de temps, de lieux, de médiations pédagogiques humaines et technologiques et de ressources. ${ }^{16}$

\section{Des angles d'analyse variés et pluridisciplinaires}

Cet enrichissement progressif du concept de FOAD se traduit par la multiplication des dimensions impliquées dans son analyse ${ }^{17}$ :

- une dimension d'ingénierie pédagogique, si on considère l'angle de la gestion, technique et pédagogique ;

- une dimension psychologique de l'apprentissage, dès l'instant où on regarde ce qui se passe du point de vue de l'autonomie, des perceptions et des spécificités de l'apprentissage ;

- une dimension technologique, prise sous l'angle des outils et ressources ;

- une dimension sociologique ou institutionnelle enfin, du point de vue des modes d'accès ${ }^{18}$.

L'importance des thématiques liées à la FOAD tend également à croître. En effet, le développement des TIC au sein de la formation conduit à poser des questions touchant à la fois à la conception d'outils, de dispositifs et de systèmes de formation, à l'assistance aux utilisateurs dans la recherche d'informations, aux médiations nécessaires et aux usages qui peuvent se développer dans des contextes d'enseignement ${ }^{19}$.

Par ailleurs, le développement des TIC interroge les organisations éducatives, quel que soit leur niveau, dans leur fonction d'administration et de gestion, de dépositaires et de distribution de l'information, d'enseignement et de recherche ou d'industrialisation de la formation. Ces questions, liées à la structure de l'offre éducative, intéressent particulièrement les sciences de l'information et de la communication.

Dans ce cadre, la question de l'intégration des dispositifs de FOAD au sein d'établissements d'enseignement supérieur peut être examinée sous l'angle de l'utilisation d'outils techniques dans la pédagogie pratiquée par les enseignants du supérieur (approche plus didactique), comme elle peut l'être sous l'angle de

16. Collectif de Chasseneuil, (2001), Accompagner les formations ouvertes, Paris, éd. L'Harmattan.

17. Les $3^{\mathrm{e}}$ Rencontres du FFFOD «La formation ouverte et à distance : l'heure des solutions mixtes », 5 et 6 mars 2002, Paris, sur le site http://www.fffod.org

18. Cf. (2002) La formation ouverte et à distance : l'heure des solutions mixtes, Actes des $3^{\text {e }}$ rencontres du FFFOD, http://www.fffod.org.fr

19. Baron G.-L., (2001), Applications et usages éducatifs des TIC,

http://www1.msh-paris.fr:8080 
l'évolution des systèmes institutionnels de formation (leur industrialisation, approche plus sociologique).

L'hétérogénéité des angles d'approche de la question n'a d'égale que la variété des analyses proposées par les diverses disciplines intéressées par ces nouveaux dispositifs. Ainsi par exemple, les travaux issus des sciences de l'éducation tendent à examiner de manière endogène et critique la question des mutations des pratiques de formations qu'implique l'usage de dispositifs de FOAD. C'est notamment la question des nouveaux métiers et compétences en formation qui peut être ainsi examinée ${ }^{20}$, ou celle des nouvelles formes d'apprentissage et des nouvelles pratiques pédagogiques qu'elles impliquent.

L'approche adoptée par les textes issus des sciences de l'information et de la communication est plus exogène. En effet, ils abordent plutôt la question sous l'optique des mutations sociales que de tels dispositifs impliquent ou génèrent. Ils étudient d'une part les usages effectifs observés ${ }^{21}$, et d'autre part l'industrialisation de la formation ${ }^{22}$ (visio-conférence, collaboration entre professions diverses, opposition entre concepteurs et utilisateurs, etc.). Ils interrogent également le positionnement des institutions face au développement de tels dispositifs.

Enfin, ce survol bibliographique ne saurait ignorer les textes issus de la communauté de ceux qui côtoient ces dispositifs ${ }^{23}$ : ce sont les chefs de projets, les enseignants impliqués dans l'élaboration de ces dispositifs ou les formateurs qui font usage de la FOAD. Ces derniers tendent à «socialiser» les dispositifs de FOAD en proposant des descriptifs détaillés, tant techniques que pédagogiques, en produisant des référentiels de compétences destinés aux formateurs ou aux enseignants, en analysant les coûts impliqués par ces environnements de formation, en soulevant des questions relatives à la normalisation et l'interopérabilité des dispositifs ${ }^{24}$. On note ainsi l'émergence d'un modèle «social» de la formation ${ }^{25}$, plus spécifiquement de «mondes sociaux de la formation », possédant leurs propres valeurs, leurs propres «figures» de formateurs et de concepteurs, leurs propres métiers. Ce modèle est destiné à compléter les approches technico-pédagogiques classiquement proposées.

20. Baron G.-L., (2001) «Usages éducatifs des technologies de l'information et de la communication: quelles nouvelles compétences pour les enseignants?» in séminaire Compétences, INRP. Alava S., (Dir) (2000), Cyberespace et formations ouvertes : vers une mutation des pratiques de formation?, éd. De Boeck.

21. Ologeanu R., (2000) Les usages de la visioconférence dans l'enseignement supérieur, http://www.educnet.education.fr/superieur/visio/visio1.htm

22. Moeglin P., (Dir.) (1998), L'industrialisation de la formation: Etat de la question. Paris, CNDP.

23. Caspar P., (Dir) (1998), Nouvelles technologies éducatives et réseaux de formation: des entreprises parlent de leurs expériences, Paris, éd. d'Organisation.

24. Cf. les études de dispositifs effectuées par l'association ALGORA sur le site: http://www.algora.org/observat/obs_pf.htm

25. Blandin B., «Les mondes sociaux de la formation », in Les TIC au service des nouveaux dispositifs de formation, Éducation permanente, $\mathrm{n}^{\circ}$ 152/2002-3. 


\section{Deux problématiques distinctes : intégration et/ou transfert de dispositif?}

Les textes abordant la question de l'intégration des TIC dans le milieu de la formation indiquent entre autres que :

- de manière générale, les TIC ne s'intègrent pas naturellement dans les établissements scolaires. De grandes différences existent entre les écoles et les disciplines ;

- l'on peut constater que les premières mises à l'épreuve en milieu éducatif de dispositifs et d'environnements technologiques sont en général réalisées alors que ces derniers ne sont encore ni techniquement stabilisés ni socialisés ;

- l'analyse des processus de «scolarisation» suivis par les différentes technologies (au sens de leur intégration progressive dans des contextes scolaires) montre que ce qui finit par se scolariser est un héritage (et non une simple transposition) d'actions de recherche et d'innovations menées en relation avec des enseignants (ou à leur initiative) longtemps auparavant ${ }^{26}$.

La question soulevée par le campus numérique $n^{\circ} 15$ met en présence deux univers distincts : celui de l'enseignement supérieur en général et celui au sein duquel le dispositif de FOAD a été conçu. Or tous deux vivent l'intégration des TIC de manière différente.

Pour les premiers, se pose le problème des nouvelles approches pédagogiques induites par l'usage des technologies. Le développement des dispositifs de FOAD suscitent des interrogations de nature didactique, tant sur les potentiels pédagogiques des environnements à développer, que sur l'évolution des activités des étudiants et enseignants, et de l'évaluation des apprentissages qu'ils engendrent.

On constate également que le développement de tels dispositifs implique une certaine «culture FOAD». Par exemple, le tutorat, qui est au cœur des dispositifs d'accompagnement ne va pas de soi dans l'enseignement supérieur, bien qu'il se développe en premier cycle universitaire. La définition de «l'intégration » proposée par les participants au campus numérique $\mathrm{n}^{\circ} 15$ est en cela significative: «L'intégration désigne le processus conduisant un établissement d'enseignement supérieur et ses acteurs à s'approprier un dispositif conçu ailleurs, donc a priori étranger à leur culture (pratiques, valeurs, etc.). » ${ }^{27}$

Par contre, les acteurs-producteurs de dispositifs de FOAD, dans le contexte spécifique de la formation continue des adultes, n'hésitent pas à évoquer la problématique du transfert ${ }^{28}$, le transfert étant entendu comme «la dissémination de

26. Baron G.-L., (2001), Usages novateurs des nouvelles technologies, http://www1.mshparis.fr:8080/

27. Collectif du Moulin (2002), Intégrer les formations ouvertes, Paris, L'Harmattan.

28. «Les usages des TIC dans la formation professionnelle : exemple de trois réseaux de formation professionnelle », ainsi que les fiches synthétiques et descriptives d'actions ou de 
l'action de formation ou du support pédagogique réalisé dans un autre lieu ou bien vers d'autres publics».

De tels transferts (à la fois du dispositif technique et pédagogique) existent et sont pratiqués au sein de la formation professionnelle. Bien que les témoignages ou documents soient rares, on note que de telles opérations ont lieu dans de petites structures et pour des niveaux de formation faible (niveau IV, par exemple: formation à la gestion-comtabilité informatisée pour les très petites entreprises ou PME du bâtiment (12 stagiaires), avec un minimum d'équipement technique.

Tenant compte de la variété des dispositifs des techniques (combinaison d'outils tels que le «chat», le courrier électronique, le forum de discussion, les plates-formes de téléformation, la visioconférence, etc.) et modèles pédagogiques (travail collaboratif, autoformation, formation tutorée, etc.) que peuvent revêtir les dispositifs de FOAD d'une manière générale, il est peut-être prématuré de se poser la question de leur transfert au sein de formations supérieures.

\section{Les expérimentations de dispositifs de FOAD dans les établissements d'enseignement supérieur}

La mise en place des projets de campus numériques implique la mutualisation de moyens tant humains, techniques qu'organisationnels, rendant ainsi l'identification des acteurs et des dispositifs de FOAD malaisée. Ce fait est clairement illustré par le nombre d'établissements impliqués dans ces projets: en janvier 2002 on note la participation de 82 universités, 22 instituts ou grandes écoles, 29 IUFM, auxquels il faut également associer la participation de 45 universités étrangères.

Les expériences prises en compte ici concernent toutes des dispositifs de FOAD actuellement opérationnels dans des formations supérieures. Ils ont recours à des environnements techniques variés (plates-formes de formation ${ }^{29}$, visioconférence, mise en ligne de ressources) et sont implantés dans des établissements d'enseignement supérieur divers - en école d'ingénieurs (Institut national polytechnique de Lorraine), au sein d'universités (université du Maine) - et sont utilisés tant dans le cadre d'une formation initiale (université de Picardie) que dans celui d'une formation continue (Institut national polytechnique de Grenoble).

dispositifs de formation utilisant les TIC, à consulter sur le site : http://www.ovarep.asso.fr/synergie/pourtous/observat/ft/reperes/utic.htm

29. Il s'agit d'un logiciel assistant la conduite des formations à distance. Il regroupe des outils nécessaires aux trois principaux utilisateurs (formateur, apprenant, administrateur) d'un dispositif qui a pour finalité la consultation à distance de contenus pédagogiques, l'individualisation de l'apprentissage et le tutorat. 
Ils ont également pour particularité d'avoir fait l'objet d'un travail d'analyse relatif aux divers usages observés (premier cycle sur mesure, PCSM) ${ }^{30}$ et visioconférence ${ }^{31}$ ) ou rendant compte des étapes nécessaires à sa mise en place (plates-formes ${ }^{32}$ ).

\section{Caractéristiques générales}

Jusqu'en 2000, rares étaient les témoignages relatifs à la mise en place et à l'usage de tels dispositifs. Ceux-ci étaient principalement le fait d'universités et s'inscrivaient dans le cadre de formations orientées vers l'univers professionnel (mise en place de DESS pour l'Université de technologie de Compiègne et l'université de Nancy 2, formation en médecine pour l'université de Paris 5, informatique et multimédia pour l'université de Picardie Jules Verne et l'université de Limoge, et chimie pour l'université du Maine).

\section{Diversité technique}

La mise en place de ces dispositifs de FOAD se caractérise par une diversité technique : visioconférence pour l'université de Marne-la-Vallée, mise en ligne de ressources dans le cadre PCSM, et plates-formes de formation. Au sein d'une même technique, telle que celle des plates-formes de formation par exemple, les choix sont hétérogènes : l'université du Mans fait reposer son dispositif de FOAD sur la plateforme WebCT, l'université de Compiègne utilise plutôt Learning Space (Lotus), l'Institut national polytechnique de Lorraine travaille sur Top Class (WBT System) et les Universités de Picardie et de Paris 5 développent leur propre plate-forme de formation.

\footnotetext{
30. Les ressources scientifiques de l'université en ligne : quels usages ?, Marseille 29-30 juin 2000, et Les Rencontres de La Rochelle des 5 et 6 juillet 2001, sur le site www.univ-enligne.prd.fr/actua.html

31. Cf. le rapport d'Ologéanu, R., ainsi que (2000), La visioconférence : usages, stratégies, moyens, rapport du GEMME, http://gemme.univ-lyon1.fr/visioconference/rapport.pdf

32. Les expériences prises en compte sont celles de :

- l'université de Picardie Jules Verne (MIAGE et DESS SIM),

- l'université du Maine (RESAFAD),

- l'Institut national polytechnique de Grenoble et l'Institut national polytechnique de Lorraine (ELAN),

- l'Institut d'administration des entreprises de Nancy (DESS CAAE),

- l'université de technologie de Compiègne (DESS DICIT),

- l'université de Limoges (DEUST conseiller pour l'intégration des TIC).
} 


\section{Diversité du public visé}

Qu'ils soient à visée internationale ou locale, destinés à des professionnels désirant se spécialiser ou des étudiants en formation initiale, les dispositifs sont utilisés auprès de publics et d'effectifs variables.

L'université du Maine par le biais de RESAFAD propose un Diplôme d'université «Communicateur multimédia à distance » à un public africain, l'INP de Grenoble s'adresse à un public de professionnels désirant se spécialiser, l'université de Limoge propose son DEUST «Conseiller pour l'intégration des TIC » à des emplois-jeunes ou aides-éducateurs, les établissements ayant recours au premier cycle sur mesure s'adressent principalement à un public d'étudiants.

\section{Diversité dans la mise en ouvre du dispositif}

Contrairement à l'usage de la visioconférence qui nécessite un environnement et une infrastructure importants et où l'enseignant gère seul la médiation des connaissances dans le cadre d'une formation, la mise en œuvre d'une FOAD reposant sur l'usage d'une plate-forme de formation nécessite généralement la mise en place d'une équipe pédagogique. Sa composition varie en termes de fonctions et d'effectifs: dans le cas du DESS CAAE de l'université de Nancy, l'équipe pédagogique est composée d'un administrateur de la plate-forme, de créateurs de cours, de tuteurs, de responsables de modules et d'une personne chargée de la médiatisation, soit au total 30 personnes pour une formation accueillant... 17 inscrits en 2000.

Ce même type d'équipe, dans le cadre de la formation déployée par l'Institut national polytechnique de Grenoble et l'Institut national polytechnique de Lorraine se compose de 62 personnes remplissant des fonctions telles que responsable de projet, responsable pédagogique, coordinateur méthodologique, concepteur, tuteur, assistant administratif et administrateur de plate-forme. La première année, cette formation a accueilli quatre élèves.

La diversité des supports technologiques utilisés dans le cadre de FOAD témoigne de l'absence d'une vision commune, au sein des établissements d'enseignement supérieur, de l'enseignement à distance. Ce manque d'homogénéité est accentué par un certain nombre de difficultés rencontrées lors de la mise en œuvre de ces dispositifs.

\section{Les principaux obstacles observés}

Les témoignages rendus mettent en perspective principalement trois difficultés :

- la première concerne la structure accueillant les dispositifs, mettant ainsi en lumière des incompatibilités structurelles à l'utilisation de tels dispositifs ; 
- la deuxième difficulté est relative aux personnes usant de tels dispositifs et met en avant l'incompatibilité des dispositifs FOAD avec les normes pédagogiques et culturelles;

- la troisième porte sur la technique, plus précisément sur la complexité de sa mise en œuvre.

\section{Incompatibilités structurelles à l'usage des dispositifs FOAD}

Les modes de reconnaissance professionnelle ne valorisent pas, semble-t-il, les efforts déployés lors de la mise en place et du fonctionnement de tels dispositifs. Les critères de promotion ou de recrutement, par exemple à l'université, restent avant tout liés à la recherche et ne prennent pas suffisamment en compte l'enseignement en général, ou l'investissement particulier que suppose l'emploi significatif des TIC. Par exemple, la quantification de certaines activités de tutorat (réponses aux messages, corrigés d'exercices), n'est pas effectuée. Cette difficulté, a priori liée aux dispositifs, masque en fait un problème de fond propre à l'enseignement supérieur : celui de la reconnaissance et de l'évaluation des activités d'enseignement et de formation.

Parmi ces obstacles, on relève des difficultés liées à la rémunération des auteurs de documents produits pour les dispositifs de FOAD, la quantification de certaines telles que le tutorat ou la production de documents, de services, la gestion des droits d'auteurs.

A cela s'ajoute le fait que la mise en place des dispositifs implique une mutualisation des compétences pédagogiques, techniques et administratives. Concrètement, cela se traduit par la nécessaire collaboration d'établissements dont le statut et l'autonomie ne les ont pas jusque-là mis en relation, ou très peu. En outre, au sein même desdits établissements, les enseignants, techniciens et personnels administratifs sont amenés à travailler plus étroitement qu'à l'ordinaire. Enfin la mise en place de dispositifs de FOAD nécessite la négociation de logiques aussi différentes que celles des concepteurs de dispositifs et celles des usagers, en l'occurrence les enseignants ${ }^{33}$. Les observations montrent que cette mutualisation des expériences individuelles et des expérimentations est nécessaire pour stabiliser les usages de tels dispositifs. En effet, des travaux mettent en valeur la différence entre d'une part les usages prescrits par le concepteur du dispositif, les usages préconisés par les initiateurs de projet, et d'autre part les usages effectifs observés sur le terrain ${ }^{34}$. Cette différence exige une négociation continue pour les faire converger.

33. Cf. l'étude d'Ologeanu R.

34. Perriault J., (1989) La logique de l'usage, Paris, Flammarion. 


\section{Incompatibilité avec les normes pédagogiques et culturelles}

La question des modèles pédagogiques véhiculés par les dispositifs et de leur compatibilité avec les modèles utilisés au sein des établissements d'enseignement supérieur se pose également. En effet, les établissements d'enseignement supérieur répondent à des spécificités qui leurs sont propres : l'autonomie pour les universités ou le rapprochement avec l'univers professionnel pour les écoles d'ingénieurs, ne sont là que des exemples. La variété de ces spécificités est à l'image de celle des modèles pédagogiques utilisés dans ces établissements. En corollaire, du point de vue de la didactique des disciplines, se pose la question de la médiatisation des disciplines : peuvent-elles toutes être «médiatisées » par des dispositifs de FOAD ?

Les normes pédagogiques en vigueur dans la FOAD, et par conséquent celles médiatisées par les dispositifs, sont principalement des normes anglo-saxonnes. Elles sont basées sur la méthode des cas (conduite de projet) et l'alternance en matière de formation ${ }^{35}$. Ces normes sont connues, voire pratiquées dans les écoles d'ingénieurs et dans la formation continue ${ }^{36}$. Or, au sein des universités sont principalement valorisés l'exposé magistral, la pédagogie déductive, où, pour prendre un raccourci, seul le concept est au cœur des choses et non le projet ou l'exemple. Il y a là une difficulté d'ordre culturel qui conditionne la rencontre de l'université et de la FOAD, et par conséquent l'appropriation et l'intégration de ces dispositifs. Cette difficulté se situe tant du côté de l'enseignant, au niveau de l'approche pédagogique, que du côté de l'étudiant, dont l'autonomie est particulièrement sollicitée par la pratique de la FOAD.

Enfin, ces dispositifs impliquent également la mise en œuvre de nouvelles compétences professionnelles. Dans le cas de la mise en ligne de ressources par exemple, ou dans le cadre plus général de l'intégration des TIC, les compétences repérées comme nécessaires aux enseignants relèvent entre autres d'une capacité ${ }^{37}$ :

- à créer, produire des outils et des services, et notamment être capable d'appréhender les spécificités des multimédias ;

- à organiser et gérer : savoir monter un projet et effectuer un cahier des charges, concevoir du matériel pédagogique et l'adapter, stimuler les processus d'autonomisation et construire les modalités d'accompagnement.

35. Comme nous l'a indiqué C. Lepineux.

36. Vinck D., (Dir) (2000), Pratiques de l'interdisciplinarité : mutations des sciences de l'industrie et de l'enseignement, PUG, Grenoble.

37. Haeuw F., Competice: outil de pilotage par les compétences des projets TICE dans l'enseignement supérieur, ALGORA, septembre 2001. 


\section{Complexité technique}

Les modèles de plate-forme, leur architecture, dépendent fortement de la nature de la formation ${ }^{38}$. Il ne semble pas exister de plate-forme «générique ». En outre, dans de nombreux cas, les modèles mis en œuvre sont polymorphes ou hybrides, ce qui les rend d'autant moins réutilisables dans le contexte d'autres formations. Tel est le cas à l'université du Maine, où chacune des quatre formations à distance repose sur une architecture spécifique.

Le travail de conception technique des enseignements dépend fortement de la flexibilité et du degré de paramétrage du dispositif. Lorsque ceux-ci sont élevés, la conception technique peut conduire à une externalisation du suivi de production des documents ou à un accroissement des équipes de travail. Par ailleurs, la gestion documentaire du dispositif accrôit le coût de conception, de même que la mise en œuvre d'une plate-forme de formation multiplie le nombre des intervenants, éclatant ainsi le rôle de l'enseignant en celui de plusieurs intervenants.

Enfin, contrairement à une idée répandue selon laquelle il n'est question que d'investissements lourds (ou matériels) en matière de dispositifs de FOAD, la principale difficulté de la formation à distance ne réside pas tant dans la construction du dispositif que dans son exploitation (et les services associés).

\section{Une conclusion, telle que l'analyse des documents recueillis permet de l'envisager}

L'enseignement à distance via internet dans l'enseignement supérieur, comme c'est souvent le cas pour les phénomènes en émergence, est caractérisé par plusieurs antagonismes importants :

- une assise conceptuelle incertaine: les bases conceptuelles du domaine, les définitions, sont variées et instables; elles dépendent notamment de l'angle pédagogique, psychologique, technologique, institutionnel - sous lequel elles sont traitées; les ébauches de réponses pluridisciplinaires apportées à la question soulevée par le campus $\mathrm{n}^{\circ} 15$ soulignent cette instabilité plus qu'elle ne la dissipe ;

- des atouts techniques inexploités: la «généricité »dans la conception des dispositifs de FOAD que l'on peut légitimement attendre comme conséquence directe de celle des TIC, dont c'est la principale caractéristique, reste pour l'heure toute théorique, puisque les dispositifs actuels semblent dépendre étroitement du type de formation dispensée ;

- une diffusion confidentielle : la capacité qu'offrent les TIC et internet à mettre potentiellement en relation un nombre d'individus très importants est battue en brèche par les effectifs particulièrement faibles observés pour les campus numériques actuellement opérationnels ;

38. Cf. les études de cas développées sur le site ALGORA : http://www.algora.org 
- une confrontation de deux univers : celui de l'enseignement supérieur d'une part, caractérisé par la primauté du conceptuel, du lien étroit unissant l'enseignant à la matière enseignée, et par un enseignement de masse peu encadré, et celui des concepteurs de dispositifs de FOAD, où démarche expérimentale et par l'exemple, standardisation des processus d'apprentissage et industrialisation sont les maîtresmots.

L'enjeu pour les principaux acteurs institutionnels ou privés est donc de contrôler le développement de la FOAD via les TIC en maîtrisant puis réduisant les antagonismes ci-dessus. De ce contrôle émergera la carte institutionnelle, économique et pédagogique de la FOAD en France. 